\title{
LA ORIENTACIÓN PARA LA AUTOGESTIÓN DEL CONOCIMIENTO EN ESTUDIANTES UNIVERSITARIOS.
}

Autor.

Belkis L. Aranda

Miembro del Centro de Estudios

Pedagógicos, Universidad de Oriente

belkisac@uo.edu.cu
Coautor.

Mayelín H. Vega

Especialista en gestión de la

información de la Universidad de Oriente
Coautor.

Belinda M. Lema.

Rectora del Instituto Superior

Tecnológico de Formación Profesional

Administrativa y Comercial.

martalema@formacion.edu.ec

País Origen: Ecuador

Recibido: 11 de Marzo del 2016

Palabras claves: Formación, Competencia, Orientación, Tecnología, Salud.

Aceptado: 15 de Abril del 2016

Key Words: Autogestión del conocimiento, biblioteca escolar, estudio independiente

\section{SUMMARY}

$\mathrm{P}$ rofessionals' formative process at the Universities establishes the necessity on consolidating students' cognitive independence due to the object of the profession it possesses. Therefore, it is essential to stimulate on searching information in different spaces, aspect which is reflected at the Study Plan of each career. It is significant the importance of the library as a scholar space who promotes and trains students in the development of the independent study, which favors the construction of knowledge. In this sense, it is the intention of this article to reflect on the theoretical and methodological foundations about knowledge's self-administration, since the scholar library conceived as a space that develops integral general culture on students.

\section{RESUMEN}

\footnotetext{
n las Universidades el proceso formativo de los profesionales establece la necesidad de consolidar la independencia cognoscitiva de los estudiantes por el objeto de la profesión que posee, por lo que es una obligatoriedad estimular la búsqueda de información en diversos espacios, aspecto que está reflejado en el Plan de Estudio de cada carrera. Se significa la importancia de la biblioteca escolar como espacio escolar que promueve y entrena a los estudiantes en el desarrollo del estudio independiente, lo que favorece la construcción del conocimiento a través de la autogestión del mismo. Sin embargo, se advierte que existen estudiantes que muestran poco desarrollo de hábitos de estudio sistemático, así como escasas habilidades para la búsqueda y construcción del conocimiento. En tal sentido, es intención de este artículo reflexionar en torno a los referentes teóricos y metodológicos acerca de la autogestión del conocimiento desde la biblioteca escolar como espacio desarrollador de la cultura general integral de los educandos.
} 


\section{INTRODUCCIÓN}

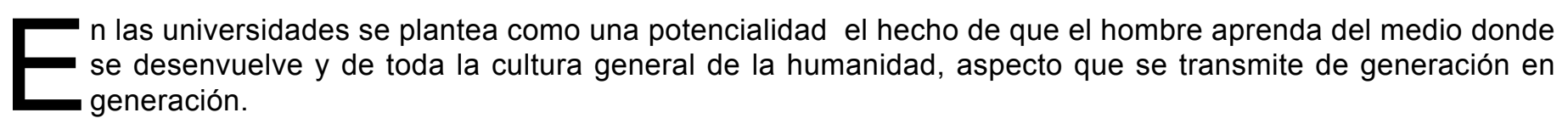

Estos conocimientos no sólo se transmiten y construyen en el espacio áulico sino en los diversos procesos formativos, lo académico, lo investigativo y lo extensionista por lo que los Centros de Información y la biblioteca escolar juegan un papel esencial.

La biblioteca escolar universitaria es un espacio que contribuye a elevar la calidad del docente, los estudiantes y trabajadores fomentando el desarrollo de la promoción de la lectura y otros procesos generadores de la cultura general integral de éstos, entrena a los estudiantes en el progreso del estudio independiente, aspecto favorecedor de la construcción del conocimiento a través de la autogestión del mismo que permite establecer una reflexión en torno a la importancia de los acciones de información de la biblioteca escolar universitaria.

La biblioteca escolar en las universidades, constituye un factor de medular importancia para la dinamización de la calidad de la labor de la escuela en el ámbito del desarrollo de una cultura general integral en estudiantes para la construcción del conocimiento, es por ello, que ésta se convierte en el centro de diseminación de la información. Además tiene el encargo de cumplir plenamente su primera gran tarea, la formación integral de las nuevas generaciones, donde su prestación de servicios debe contribuir a elevar la calidad de la enseñanza en el currículo de los estudiantes universitarios.

En tal sentido, es intención de este trabajo reflexionar en torno a algunas consideraciones teóricas y metodológicas para favorecer la autogestión del conocimiento desde la biblioteca escolar como espacio para contribuir al desarrollo de la cultura integral de los estudiantes universitarios.

\section{DESARROLLO}

La adquisición del conocimiento está en los medios intelectuales del hombre, a medida que crece el conocimiento de éste se da un cambio cualitativo debido al incremento de reorganización del conjunto y adquisición de los mismos, que se sustenta además por el modo de conocer los aspectos de la realidad que abarca desde su reflejo superficial hasta el dominio de las leyes que rigen sus fenómenos.

Percibir es la actividad mental mediante la cual llegan al cerebro los estímulos del exterior y se realiza el proceso de cognición. De otro lado, concebir es la actividad mental mediante la cual resultan conceptos e ideas a partir de los estímulos percibidos, los cuales determinan a su vez los conceptos de entender y comprender que hacen que el proceso cognoscitivo culmine en aprendizaje. Se debe diferenciar el entender de comprender, se entiende un hecho, una relación, una palabra, un método, en cambio, se comprende una serie, un sistema, un plan. La comprensión es una aptitud elevada del pensamiento humano.

El conocimiento puede definirse como aquella información almacenada en una entidad y que puede ser utilizada por la inteligencia de acuerdo a ciertos objetivos. Se puede dividir el conocimiento en dos grupos, uno natural que pertenece a los organismos vivos con sistema nervioso y el otro, artificial, que poseen aquellos mecanismos que simulen o reproducen parcialmente al sistema natural. En el caso del hombre, el conocimiento es producto de procesos mentales que parten de la percepción, el razonamiento o la intuición. En ese sentido, uno de los conceptos fundamentales para el conocimiento es la capacidad de relación o asociación entre estos.

Según Puleo (1985), el conocimiento como información específica acerca de algo puede referirse a dos entidades diferentes, su forma y su contenido. La forma es esencial al determinar las condiciones por las cuales algo puede llegar a ser objeto del conocimiento. El contenido se produce bajo influencias externas y donde se $\mathrm{n}$ distinguir dos actividades de la mente: percibir y concebir.

Se rescata la idea de que la información es el insumo esencial del conocimiento, en ese sentido el desarrollo de las redes de información fundamentadas sobre todo por la tecnología de Internet crea un nuevo contexto a las organizaciones de hoy. En este entorno, las posibilidades de publicar, almacenar y distribuir la información se ha expandido considerablemente, conduciendo a las organizaciones al planteamiento de objetivos más ambiciosos y, poco a poco, al traslado de la idea de gerencia de la información a la idea de gerencia del conocimiento.

El conocimiento se refiere a las características internas y al comportamiento de un sujeto, tanto en las reacciones como en sus relaciones con los estímulos del ambiente. Se refiere a la diferenciación perfecta con relación a otras cosas que pudieran parecer iguales, es todo lo que un ser humano ha aprendido, aplicado y organizado, supone una reflexión en correspondencia con la información asimilada. La información es el conocimiento transformado, 
su forma representa dicho conocimiento, asimismo, el conocimiento tiene las propiedades de comunicar, revelar, manifestar y de recomendar las determinaciones para actuar de forma productiva.

Dentro de sus objetivos, la biblioteca escolar universitaria es la encargada de informar, orientar, desarrollar variadas actividades que conlleven a profundizar en un caudal de conocimientos contribuyendo al desarrollo de su cultura. Se caracteriza por promover los recursos humanos que intervienen en los procesos de formación general de habilidades, capacidades y cualidades personales para la construcción del conocimiento, para ello este espacio gestor de información debe atemperarse al Plan de Estudio de todas las especialidades que se desarrollan en la universidad de manera que las actividades formativas que en ella se desarrollan se corresponda con el contenido del plan de estudio, incluyendo los cursos optativos, electivos y facultativos.

La principal característica funcional del sistema de gestión del conocimiento es hacer coincidir las necesidades concretas de información de las distintas personas y equipos de trabajo con la disponibilidad efectiva de dicha información.

La gestión del conocimiento es un instrumento básico que permite la formación profesional del docente, identificar, encontrar, clasificar, proyectar, presentar y usar de un modo más eficiente el conocimiento y la experiencia que poseen los estudiantes de las carreras pedagógicas de las universidades.Este importante elemento -la gestión del conocimiento - desde la biblioteca escolar convoca a determinar las ideas, incrementarlas y explotarlas para ganar capacidad profesional, impulsa a comprender el conocimiento aumenta los niveles de información y crea un nuevo valor para el estudio y la apropiación de los diferentes contenidos de modo que éstos sean capaces de enfrentar desde los problemas más simples hasta los más complejos de la vida.

Se entiende la gestión como la acción y efecto de integrar recursos para el desarrollo eficiente y eficaz de los procesos organizativos, según Stoner (1996) son las actividades coordinadas para dirigir y controlar una organización o proceso. Son procesos de la gestión: planificar, organizar, dirigir y controlar. El concepto de conocimiento es ampliamente estudiado en la Teoría del Conocimiento que inicia su construcción en Grecia Antigua y se continúa construyendo, matizada por el desarrollo de diferentes corrientes del pensamiento. Este concepto se estudia también desde diferentes puntos de vista. Así, conocimiento puede ser entendido con relación a datos e información personalizada, con relación al estado de la mente como estado de conocer y comprender. Puede ser definido también como objetos que son almacenados y manipulados, proceso de aplicación de la experiencia, condición de acceso a la información y potencial que influye en la acción. La gestión del conocimiento ha sido definida por varios autores. Wiggs (citado por Artiles Visval, 2005), plantea que ésta "hace énfasis en facilitar y gestionar actividades relacionadas con el conocimiento tales como la creación, captura, transformación y uso. Su función es planificar, implementar, operar y gestionar todas las actividades relacionadas con el conocimiento y los programas requeridos para la gestión efectiva del capital intelectual".

Por otra parte, Leticia y Sara Artiles Visval (2005) plantean que la gestión del conocimiento "Es el sistema que organizan los flujos de información, externos e internos, propicia la generación, apropiación, intercambio y uso de conocimientos necesarios para el incremento de la eficiencia y calidad en los procesos fundamentales de las organizaciones"; Estrada y Febles (2002), consideran que es un "proceso se añade valor a los conocimientos existentes y se generan nuevos conocimientos científicos, un nuevo mercado y nuevos servicios " destacando que consideran la gestión del conocimiento un "proceso".

Sveiby (2005), señala que la gestión del conocimiento "Se enfoca en convertir el conocimiento individual en conocimiento organizacional para la aplicación de procesos sistemáticos y tecnológicos para identificar, dirigir y diseminar el conocimiento requerido para apoyar a la resolución de problemas decisivos, asegurando que los decisores tengan acceso al conocimiento requerido, en un formato que cree sentido a ellos".

La autogestión del conocimiento de los estudiantes de universitarios permite traducir el conocimiento en acción y éste en resultados, los objetivos de la gestión del conocimiento son:

- Incrementar las oportunidades del desarrollo intelectual.

- Aumentar la comunicación.

- Aumentar el conocimiento de la profesión

- Elevar el liderazgo y protagonismo en el desarrollo de tareas integradoras, trabajos extracurriculares, de curso y de diploma.

- $\quad$ Elevar el rendimiento académico. 
La autogestión del conocimiento hace referencia a herramientas que proponen dirigir y controlar el conjunto de procesos productivos que utilizan la orientación y la información o conocimiento como factor fundamental para generar un aprendizaje desarrollador en los estudiantes.

En este ámbito es esencial la atención y el estudio de los procesos de transmisión de conocimientos vinculados al ciclo de vida de la información: las formas de crear, adquirir, distribuir, usar, almacenar y revisar información o conocimiento.

La autogestión desde una correcta orientación para el estudiante, activa el conocimiento, implica la construcción del mismo, brinda objetivos para convertir el conocimiento individual en pertinencia para compartir de manera grupal, o sea, para incrementar su esencia intelectual, pues la finalidad última de esta herramienta es, hacer operativo y eficiente el saber almacenado en los estudiantes, lo que supone, además, evaluar y considerar su intelecto.

El perfil de los usuarios cambia en forma radical y la biblioteca escolar universitaria para poder enfrentar las nuevas necesidades de su estudiantado conlleva a la instrumentación de nuevos modelos de gestión. El no hacerlo generará que dicha biblioteca pierda número de asistencia por parte de todos los usuarios y su relevancia en los procesos de docencia, difusión de la cultura e investigación.

Con una adecuada orientación educativa podemos constatar que se logra una acertada autogestión del conocimiento de los estudiantes de carreras pedagógicas que permite convertir este conocimiento en una difícil labor obteniendo óptimos resultados, se precisa además que los objetivos de la gestión del conocimiento están destinados a aumentar las oportunidades del desarrollo intelectual, la comunicación y el conocimiento de la profesión, así como elevar su rendimiento académico y el protagonismo y liderazgo a mantener en el desarrollo de tareas integradoras, trabajos extracurriculares, de curso y de diploma.

En general de manera explícita se establece la necesidad de lograr con una precisa orientación educativa facilitar la autogestión del conocimiento en los estudiantes universitarios ya que esto ayudará considerablemente el crecimiento personal y profesional en el proceso de formación inicial de los mismos, permitirá el perfeccionamiento y la rapidez en sus habilidades profesionales que causarán impacto en las acciones que implementan en el ejercicio de las funciones profesionales en el proceso de práctica laboral y fomentará la identidad profesional pedagógica que se conforma en la excelencia en la dirección de los procesos que los que estos se insertan.

\section{CONCLUSIONES}

Las naciones y el hombre se distinguirán cada vez por su cultura, aquellas que exploten el conocimiento acumulado a la hora de innovar o resolver algún problema para hacer las cosas bien y de forma rápida, se convertirán en una universidad atemperada y promotora de saberes que forma profesionales inteligentes.

El conocimiento, como la clave fundamental para un futuro exitoso, es un movimiento que nace en el interior de las bibliotecas escolares universitarias integrantes de la organización aprenden tanto de los errores como de los éxitos propios y ajenos. Este movimiento basado en el conocimiento está cambiando los enfoques sobre cómo una organización comienza, progresa y madura, muere o se reforma, en él no sólo se incluye el conocimiento que los profesionales, docentes en formación y en ejercicio que poseen sobre un tema, sino también la cultura general de los integrantes de la Universidad de Ciencias Pedagógicas. 


\section{BIBLIOGRAFÍA}

Artiles Visval, L. (2005). Gestión del conocimiento y cooperación internacional en el ámbito universitario. Curso Taller, Gestión del Conocimiento para la cooperación y movilización de recursos en ambiente académico (Formato Digital), Lima, 23 - 26 de mayo de 2005.

Canals, A. (2003). La gestión del conocimiento. EN Acto de presentación del libro Gestión del Conocimiento. Barcelona (en línea) UOC. (Consulta: 6 de noviembre de 2008), Disponible en UOC.

Estrada Sentí, V. y J. P. Febles Rodríguez (2002): Gestión del Conocimiento en la Educación Superior. En: Las Nuevas Tecnologías de la Información y la Comunicación aplicadas a la Educación Superior. (monografía en CD ROM), Editorial Feijoo, Villa Clara.

Lozano Quince, Mm., A. Sevilla Escribano y R. Valtueña Rincón (2000). Aproximación a la gestión del conocimiento. Una visión práctica (en línea). Disponible en: administraciónelectrónica.gob.es. Consultado el 3 de agosto del 2013.

Ponjúan Dante, G. (2006). Introducción a la Gestión del Conocimiento. La Habana: Editorial Félix Varela.

Rosental, M. y P. Ludin (1973). Diccionario filosófico. Editora Política: La Habana, 1973. 